\title{
Tireotoxicose Induzida pela Amiodarona - Revisão de Literatura e Atualização Clínica
}

\author{
Amiodarone-Induced Thyrotoxicosis - Literature Review \& Clinical Update
}

Luciana Vergara Ferraz de Souza, ${ }^{\circledR}$ Maria Thereza Campagnolo, ${ }^{2}$ Luiz Claudio Behrmann Martins, ${ }^{3}$ Maurício

Ibrahim Scanavacca ${ }^{3}$

Faculdade de Medicina de Jundiaí, ${ }^{1}$ Jundiaí, SP - Brasil

Centro Universitário Lusiada Faculdade de Ciências Médicas de Santos, ${ }^{2}$ Santos, SP - Brasil

Universidade de São Paulo Instituto do Coração - Arrritmia e Marcapasso, ${ }^{3}$ São Paulo, SP - Brasil

\section{Resumo}

A amiodarona é amplamente utilizada no tratamento de arritmias atriais e ventriculares, porém devido sua alta concentração de iodo, o uso crônico da droga pode induzir distúrbios tireoidianos. A tireotoxicose induzida pela amiodarona (TIA) pode descompensar e exacerbar anormalidades cardíacas subjacentes, provocando aumento da morbidade e mortalidade, principalmente em pacientes com fração de ejeção do ventrículo esquerdo $<30 \%$.

Os casos de TIA são classificados em dois subtipos que direcionam a conduta terapêutica. Os riscos e benefícios de manter a amiodarona devem ser avaliados de maneira individualizada, e a decisão de continuar ou suspender a droga deve ser tomada conjuntamente por cardiologistas e endocrinologistas.

O tratamento de TIA tipo 1 é semelhante ao do hipertireoidismo espontâneo, sendo indicado o uso de drogas antitireoidianas (metimazol e propiltiouracil) em doses elevadas. A TIA tipo 1 mostra-se mais complicada, pois apresenta proporcionalmente maiores números de recorrências ou até mesmo a não remissão do quadro, sendo recomendado o tratamento definitivo (tireoidectomia total ou radioiodo).

TIA tipo 2 é geralmente autolimitada, mas devido a elevada mortalidade associada a tireotoxicose em pacientes cardiopatas, o tratamento deve ser instituído para que o eutireoidismo seja atingido mais rapidamente. Em casos bem definidos de TIA tipo 2, o tratamento com corticosteroides é mais efetivo do que o tratamento com drogas antitireoidianas.

Em casos graves, independentemente do subtipo, a restauração imediata do eutiroidismo por meio da

\section{Palavras-chave}

Amiodarona/uso terapêutico; Arritmias Cardíacas; Iodo; Hipertireoidismo; Tireotoxicose; Hipotireoidismo; Tireotoxicose; Tireoidite.

Correspondência: Luciana Vergara Ferraz de Souza •

Faculdade de Medicina de Jundiaí - R. Francisco Teles, 250. CEP 13202-550,

Vila Arens, II Jundiaí, SP - Brasil

E-mail: luciv.f@gmail.com

Artigo recebido em 06/11/2019, revisado em 16/09/2020, aceito em $04 / 11 / 2020$

DOI: https://doi.org/10.36660/abc.20190757 tireoidectomia total deve ser considerada antes que o paciente evolua com piora clínica excessiva, pois a demora na indicação da cirurgia está associada ao aumento da mortalidade.

\section{Introdução}

Amiodarona é uma droga antiarrítmica classe III frequentemente utilizada no tratamento de arritmias atriais e ventriculares, ${ }^{1}$ principalmente quando são refratárias a outras drogas antiarrítmicas. ${ }^{2}$ É também utilizada na profilaxia da morte súbita de causa cardíaca em pacientes de alto risco, sobretudo em pacientes sem acesso ao cardioversor desfibrilador implantável, apresentando redução da mortalidade quando comparada a placebo e outros antiarrítmicos. $^{3}$

Devido à sua alta concentração de iodo, a amiodarona pode induzir disfunção tireoidiana (hipertireoidismo ou hipotireoidismo) em até $36 \%$ dos pacientes que fazem uso crônico desta medicação. ${ }^{4,5} \mathrm{~A}$ incidência de hipertireoidismo varia de $2 \%$ a $18 \%,{ }^{4-12}$ e a de hipotireoidismo de $5 \%-22 \%$ (Tabela 1). ${ }^{4-10,12} \mathrm{~A}$ influência do iodo no desenvolvimento desses distúrbios tireoidianos é tamanha que, de acordo com seu consumo alimentar regional, percebe-se uma mudança na forma como a amiodarona altera o comportamento da tireoide. Proporcionalmente, nas áreas onde o consumo de iodo é elevado predominam os casos de hipotireoidismo induzido pela amiodarona, enquanto em locais de baixa ingestão a incidência de tireotoxicose induzida pela amiodarona (TIA) é maior., ${ }^{4,6,8}$

O hipotireoidismo induzido pela amiodarona é de menor gravidade que o hipertireoidismo e tem tratamento mais simples. Nos casos de hipotireoidismo não é necessária a retirada da amiodarona, e o tratamento pode ser feito apenas com a introdução da levotiroxina. Em alguns casos subclínicos o ajuste (redução) da dosagem da amiodarona pode ser suficiente para que ocorra a normalização da função tireoidiana. Portanto, nos pacientes subclínicos não é necessária a reposição hormonal, apenas a análise regular da função tireoidiana para avaliar a progressão para hipotireoidismo. ${ }^{13,14}$

Clinicamente, quadros de TIA oferecem riscos maiores de complicações, além do diagnóstico e tratamento serem muito mais complexos. A exposição prolongada a altos níveis de hormônios tireoidianos pode levar ao aparecimento de arritmias e a uma rápida deterioração da função cardíaca. 5,15 


\begin{tabular}{|c|c|c|c|c|c|}
\hline Primeiro autor, Ano & País & Número de pacientes & $\begin{array}{l}\text { Hipotireoidismo } \\
\text { induzido pela } \\
\text { amiodarona }\end{array}$ & $\begin{array}{l}\text { Tireotoxicose induzida } \\
\text { pela amiodarona }\end{array}$ & Tipo de estudo \\
\hline $\begin{array}{l}\text { Martino } E,^{8} \\
1984\end{array}$ & $\begin{array}{l}\text { Itália } \\
\text { E.U.A. }\end{array}$ & $\begin{array}{l}\text { Itália: } 188 \\
\text { E.U.A.: } 41\end{array}$ & $\begin{array}{c}\text { Itália } 10(5 \%) \\
\text { E.U.A.: } 9(22 \%)\end{array}$ & $\begin{array}{l}\text { Itália: } 18(9.6 \%) \\
\text { E.U.A.: } 1(2 \%)\end{array}$ & Não descrito \\
\hline $\begin{array}{l}\text { Trip MD, }{ }^{4} \\
1991\end{array}$ & Países Baixos & 58 & $10(17,2 \%)$ & $11(18,9 \%)$ & Prospectivo \\
\hline $\begin{array}{l}\text { Yiu KH, } \\
2009\end{array}$ & Hong Kong & 354 & $73(20.6 \%)$ & $\begin{array}{c}57(16.1 \%) \\
\text { TIA 1: } 5 / 57 \\
\text { TIA 2: } 13 / 57 \\
\text { Mista/incerta: } 35 / 57\end{array}$ & $\begin{array}{c}\text { Prospectivo } \\
2000-2005\end{array}$ \\
\hline $\begin{array}{l}\text { Stan } M N,{ }^{25} \\
2013\end{array}$ & E.U.A. & 169 & Não estudado & $\begin{array}{c}23(13,6 \%) \\
\text { TIA } 1: 7 / 23 \\
\text { TIA 2: } 13 / 23 \\
\text { TIA mista/incerta: } 3 / 23\end{array}$ & $\begin{array}{c}\text { Retrospectivo } \\
\text { 1987-2009 } \\
\text { Adultos com doença } \\
\text { cardíaca congênita }\end{array}$ \\
\hline $\begin{array}{l}\text { Huang C-J, }{ }^{12} \\
2014\end{array}$ & Taiwan & 527 & $69(13.1 \%)$ & $21(4 \%)$ & $\begin{array}{l}\text { Retrospectivo } \\
2008-2009\end{array}$ \\
\hline $\begin{array}{l}\text { Uchida T, }{ }^{11} \\
2014\end{array}$ & Japão & 225 & Não estudado & $13(5.8 \%)$ TIA 2 & $\begin{array}{l}\text { Retrospectivo } \\
2008-2012\end{array}$ \\
\hline $\begin{array}{l}\text { Lee KF, } \\
2010\end{array}$ & Hong Kong & 390 & $87(22 \%)$ & $24(6 \%)$ & $\begin{array}{l}\text { Retrospectivo } \\
2005-2007\end{array}$ \\
\hline $\begin{array}{l}\text { Benjamens S, } \\
2017\end{array}$ & Países Baixos & 303 & $33(10,8 \%)$ & $44(15,5 \%)$ & $\begin{array}{c}\text { Retrospectivo } \\
1984-2007\end{array}$ \\
\hline $\begin{array}{l}\text { Barrett B, }{ }^{10} \\
2019\end{array}$ & E.U.A. & 190 & $26(13.7 \%)$ & $\begin{array}{c}4(2.1 \%) \\
25 \% \text { resolução } \\
\text { espontânea }\end{array}$ & $\begin{array}{c}\text { Retrospectivo } \\
2007-2018 \\
\text { Crianças e adultos } \\
\text { jovens }\end{array}$ \\
\hline
\end{tabular}

Um estudo observacional analisando 354 pacientes em uso crônico de amiodarona demonstrou um aumento significativo de eventos cardiovasculares maiores no grupo que desenvolveu TIA, comparado ao grupo que permaneceu eutireoideo (31,6\% vs. 10,7\%, p<0.01), especialmente devido à alta incidência de arritmias ventriculares com necessidade de hospitalização (7\% vs. 1,3\%, p = 0.03). ${ }^{5}$ Outro estudo relatou uma taxa de mortalidade de $10 \%$ antes do controle da tireotoxicose, associada a fração de ejeção do ventrículo esquerdo (FEVE) $<30 \%$. $^{15}$

Com base em estudos e diretrizes recentes, foram revisados e sintetizados de forma prática os principais aspectos diagnósticos e terapêuticos da TIA. Também, destaca-se a importância das decisões terapêuticas a serem tomadas em conjunto entre cardiologistas e endocrinologistas.

\section{Métodos}

Foi realizada uma revisão da literatura através de busca na MEDLINE utilizando as combinações dos termos MeSH: "Amiodarone", "Thyrotoxicosis" e "Thyroid". Também foram feitas buscas manuais e eletrônicas de referências citadas nos estudos avaliados. Foram incluídos estudos clínicos que abordam alterações tireoidianas secundárias ao uso da amiodarona, com foco na incidência e no tratamento clínico e cirúrgico. Foram excluídos os trabalhos que abordam transtornos causados pela amiodarona em outros órgãos e relatos de caso com número inferior a dez pacientes. Nos dados compilados também foram analisados os consensos mais atuais da Sociedade Brasileira de Endocrinologia e Metabologia (SBEM), Associação Americana de Tireoide (ATA) e Associação Europeia de Tireoide (ETA).

\section{Amiodarona: mecanismo de ação sobre a tireoide}

A amiodarona pode agir influenciando a glândula tireoide de diversas maneiras. Estruturalmente, a amiodarona é uma medicação diiodinada, sendo $37 \%$ do seu peso molecular referente ao iodo, portanto a cada $200 \mathrm{mg}$ de amiodarona (dose diária de manutenção) cerca de $7.5 \mathrm{mg}$ de iodo são liberadas. A dose diária de iodo recomendada pela Organização Mundial de Saúde é de 0,15mg (adultos), ${ }^{16}$ e com o uso da amiodarona, cerca de 7,5mg de iodo é liberado na forma livre no organismo diariamente, excedendo a dose recomendada em 50 vezes. ${ }^{17}$

A medicação também possui extrema similaridade com os hormônios triiodotironina (T3) e tiroxina (T4), ${ }^{18}$ e sua longa meia-vida garante a permanência da substância no organismo por até 100 dias, o que potencializa sua toxicidade e permite que os efeitos colaterais ocorram durante o uso e mesmo após a suspensão do medicamento. ${ }^{19-21}$

Apesar do reconhecimento de que a medicação influencia a tireoide propriamente dita e a metabolização de seus hormônios no organismo, ainda são escassas informações a respeito do seu mecanismo de ação. A inibição da enzima 5'-deiodinase é uma das teorias sobre a forma pela qual a amiodarona age sobre o metabolismo dos hormônios tireoidianos. Essa interação resulta em aumento sérico 
do T3 reverso e T4, substratos da enzima em questão, concomitantemente com a diminuição do T3, produto da conversão realizada pela molécula inibida. A sobrecarga de iodo e a citotoxicidade induzidas pelo medicamento também corroboram a explicação do surgimento de distúrbios tireoidianos como efeitos colaterais do uso crônico da medicação. ${ }^{18,22}$

\section{Tireotoxicose induzida pela amiodarona (TIA)}

A TIA está associada a altas taxas de eventos cardiovasculares maiores e ao aumento da mortalidade, principalmente da morte cardiovascular. O surgimento ou recorrência de arritmias ventriculares e a disfunção ventricular esquerda severa (FEVE $<30 \%$ ) são os principais fatores relacionados a este aumento. ${ }^{5,14,15}$ Portanto, a restauração do eutireoidismo deve ser estabelecida o quanto antes, e em casos emergenciais a tireoidectomia pode ser indicada para uma resolução rápida da tireotoxicose. . 14,19,23,24 $^{2}$

Os casos de TIA são divididos em dois subtipos devido a diferenças na fisiopatologia e a necessidade de tratamento direcionado. A TIA tipo 1 (TIA 1) tem lugar por meio da produção autônoma de hormônios tireoidianas devido à sobrecarga de iodo, particularmente concomitante às alterações prévias da tireoide (nódulos tireoidianos ou doença de Graves latente). A TIA tipo 2 (TIA 2) é a forma mais frequente e ocorre em pacientes com a tireoide previamente saudável, correspondendo a uma tireoidite destrutiva devido a citotoxicidade direta da amiodarona nas células foliculares, com consequente liberação das reservas hormonais pré-formadas e indução da tireotoxicose. ${ }^{11,14,19,24,25}$ A figura 1 ilustra as diferenças na fisiopatologia dos dois subtipos. Ocasionalmente essa distinção é complicada essa distinção é complicada e existe sobreposição dos dois subtipos, e esses casos são denominados como formas mistas ou indefinidas. . $^{13,14,19}$

\section{Diagnóstico}

Os meios para a identificação de pacientes sob maior risco de desenvolverem disfunção tireoidiana secundária ao uso da amiodarona ainda não foram definidos. ${ }^{14,26} \mathrm{~A}$ Associação Americana de Tireoide recomenda avaliação da função tireoidiana por meio da dosagem sérica de tireotropina (TSH) e T4 livre. A função tireoidiana deve ser avaliada antes de iniciar a amiodarona, dentro dos primeiros três meses após o início da droga, e posteriormente a cada três a seis meses. ${ }^{24}$
Inicialmente, pacientes tratados com amiodarona apresentam alterações da função tireoidiana, porém a maioria retorna à normalidade sem a necessidade de tratamento ou descontinuação da droga. Nos três primeiros meses de tratamento com amiodarona, ocorre aumento dos níveis séricos de TSH, T4 e T3 reverso, e concomitante diminuição de T3. Posteriormente, ocorre normalização dos níveis de TSH, T4 e T3, podendo o T4 manter-se no limite superior de normalidade ou discretamente elevado, e o T3 reverso permanecer elevado. ${ }^{14}$

A dosagem de TSH é o método mais sensível e específico para o diagnóstico de hipertireoidismo, pois pequenas alterações nos níveis de T4 livre provocam mudanças expressivas nas concentrações de TSH. No hipertireoidismo subclínico, os níveis de TSH encontram-se baixos ou até indetectáveis, e os valores de T4 livre e T3 apresentam-se normais. ${ }^{24} \mathrm{Na}$ tireotoxicose, o TSH encontra-se muito baixo ou indetectável, e os níveis de T4 livre e/ou T3 elevados. ${ }^{19,24}$

Os pacientes com TIA podem ser assintomáticos ou apresentar um quadro clínico típico de hipertireoidismo, como sintomas de palpitações, tremores, sudorese, intolerância ao calor, nervosismo e perda de peso. O bloqueio beta adrenérgico da amiodarona no coração pode justificar a ausência de palpitações, o que torna a apresentação clínica de TIA ainda mais insidiosa. ${ }^{17} \mathrm{O}$ diagnóstico de tireotoxicose é confirmado pelos níveis séricos de TSH suprimidos e níveis elevados dos hormônios tireoidianos T3 e T4 livres. ${ }^{14,19}$

A diferenciação entre os dois subtipos de TIA pode ser difícil, no entanto, alguns parâmetros laboratoriais associados à ultrassonografia de tireoide com Dopplerfluxometria podem ser usados para a distinção apropriada. ${ }^{13,14,19,24}$ As características dos subtipos de TIA estão sintetizadas na Tabela 2.

Acreditava-se que o nível sérico de interleucina-6 se apresentava altamente elevado nos casos de TIA 2 e assim seria útil na diferenciação dos subtipos de TIA, porém há uma sobreposição entre os subtipos e, portanto, não pode ser utilizado para a distinção. ${ }^{24,27}$ A captação de radioiodo $\left({ }^{131} \mathrm{I}\right.$ ou ${ }^{123}$ I) é útil nessa diferenciação em áreas de baixa ingestão de iodo, pois nessas regiões os pacientes com TIA 2 apresentam captação suprimida de radioiodo. Na TIA 1 a captação pode ser baixa, normal ou até elevada. Todavia, em áreas com ingestão suficiente de iodo, caso da maior parte das regiões

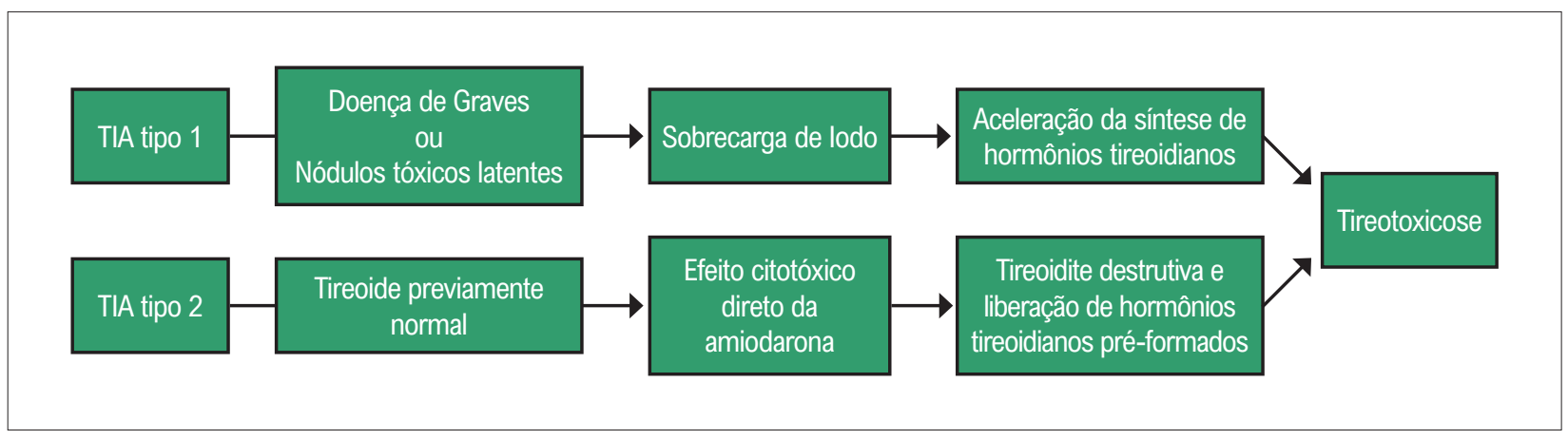

Figura 1 - Fisiopatologia das principais formas de TIA. TIA: tireotoxicose induzida pela amiodarona. 


\begin{tabular}{|c|c|c|}
\hline Características & TIA tipo 1 & TIA tipo 2 \\
\hline Alterações tireoidianas prévias & Sim & Usualmente não \\
\hline Doppler fluxometria & Vascularidade aumentada & Ausência de hipervascularidade \\
\hline Captação de iodo radioativo & Baixa, normal ou elevada & Suprimida \\
\hline Anticorpos antitireoidianos & Presente se relacionado a doença de Graves & Usualmente ausentes \\
\hline Início após amiodarona & Curto (média de 3 meses) & Longo (média de 30 meses) \\
\hline Remissão espontânea & Não & Possível \\
\hline Evolução para hipotireoidismo & Não & Possível \\
\hline Tratamento de primeira linha & Drogas antitireoidianas & Glicocortocoides orais \\
\hline Tratamento definitivo subsequente & Geralmente sim & Não \\
\hline
\end{tabular}

Modificado de Bartalena L et al., ${ }^{14}$ TIA: tireotoxicose induzida pela amiodarona.

metropolitanas do Brasil, a captação de radioiodo encontra-se sempre suprimida tornando a investigação inútil. ${ }^{14,24,28}$

A detecção de anticorpos anti-tireoperoxidase (anti-TPO) $)^{14,24}$ e a presença de bócios difusos ou nodulares na ultrassonografia de tireoide apontam para TIA 1, ${ }^{21,23}$ contudo devido a sua elevada prevalência na população esses achados também não excluem a TIA 2. ${ }^{13,14,24}$ Diversos estudos recentes indicam que a ausência de hiperfluxo na dopplerfluxometria é sugestiva de TIA 2. ${ }^{19,24,27,28}$ Estes achados não devem ser utilizados de forma isolada devido a possibilidade de formas mistas. ${ }^{14}$

\section{Manter ou suspender a amiodarona?}

A necessidade da retirada da amiodarona ainda é controversa. Em muitos casos, ela é a única medicação capaz de controlar a arritmia cardíaca, e devido a sua meia-vida prolongada, a retirada não traria benefícios imediatos. ${ }^{14}$ Além disso, é importante salientar que alguns pacientes possuem recorrência dos distúrbios tireoidianos, mesmo meses após a suspensão de amiodarona. Ademais, a droga apresenta propriedades antagonistas de T3 e inibe a conversão de T4 para T3 no coração, portanto a sua suspensão poderia agravar as manifestações clínicas. ${ }^{20,21,24}$

A TIA 2 é geralmente autolimitada, e a amiodarona pode ser mantida nesses pacientes. ${ }^{14,29-32}$ Estudos observacionais com pacientes TIA 2 revelaram que os pacientes retornam ao eutireoidismo mesmo mantendo a amiodarona. ${ }^{29,30,31}$ No entanto, estudos mostram uma variação de $8 \%$ a $73 \%$ de recorrência da tireotoxicose em pacientes que mantiveram o uso da medicação. ${ }^{29,31,33,34} \mathrm{Um}$ estudo com 10 anos de seguimento envolvendo 50 pacientes que mantiveram amiodarona apresentou apenas três casos de recorrência da tireotoxicose, sendo muito mais brandos do que no primeiro episódio. ${ }^{32}$

A decisão de retirar a amiodarona deve ser individualizada e tomada em conjunto pelo cardiologista e pelo endocrinologista, levando em consideração os riscos e benefícios da retirada da droga. ${ }^{14,19,24}$ É amplamente aceito que se continue a medicação em pacientes críticos com arritmias que ameaçam a vida e que apresentem boa resposta cardíaca a droga. 14,24,32 Se as condições cardíacas estiverem estáveis e houver uma alternativa segura, a amiodarona pode ser descontinuada. ${ }^{13,14}$

\section{Tratamento}

Em pacientes clinicamente estáveis com evidência que diferencia o subtipo, o tratamento deve ser estabelecido de acordo com o subtipo no qual o paciente se encaixa. ${ }^{14,19,24}$ Nos quadros de tireotoxicose moderada com comprometimento da função cardíaca, a Associação Americana de Tireoide recomenda iniciar terapia combinada com drogas antitireoidianas e corticosteroides. ${ }^{24}$

Se o paciente apresentar deterioração rápida da função cardíaca, tireoidectomia de emergência deve ser feita independentemente do subtipo TIA. ${ }^{14,24}$ A Figura 2 mostra o algoritmo para manejo de TIA conforme proposto pela Associação Europeia de Tireoide. ${ }^{14}$ Como nos casos de TIA a tireoide encontra-se repleta de iodo, o tratamento com iodo radioativo é inviável por pelo menos seis a nove meses a partir da suspensão da droga. ${ }^{13,19,20}$

\section{Tratamento: TIA 1}

O tratamento de TIA 1 é feito com drogas antitireoidianas (DAT), mas estas são menos efetivas devido à alta concentração de iodo, e se faz necessário o uso de doses maiores (40-60mg/dia de metimazol ou doses equivalentes de propiltiouracil). ${ }^{14,24}$ Se o paciente permanecer estável, a DAT deve ser mantida até a restauração do eutireoidismo, ${ }^{14,19,20,35}$ geralmente entre três e seis meses. ${ }^{24}$

O perclorato de potássio pode ser associado nas primeiras semanas para diminuir a captação de iodo pela tireoide e tornar a tireoide mais sensível as DAT. 1,14,19,35,36 Devido a sua toxicidade, este não deve exceder $1 \mathrm{~g} / \mathrm{dia}$ e não deve ser mantido por mais de 4-6 semanas.,14

A tireotoxicose pode recorrer ou até não entrar em remissão, e nesses casos o tratamento definitivo é recomendado. ${ }^{14,19,36}$ Se a amiodarona for descontinuada, o tratamento definitivo com radioiodo pode ser feito após seis a nove meses. A tireoidectomia deve ser considerada se não for possível a retirada da amiodarona. ${ }^{14,19}$ De uma forma geral, o tratamento definitivo de TIA 1 é semelhante ao do hipertireoidismo espontâneo. ${ }^{14}$

\section{Tratamento: TIA 2}

A TIA 2 é geralmente autolimitada, entretanto devido ao aumento da mortalidade associada a tireotoxicose em 


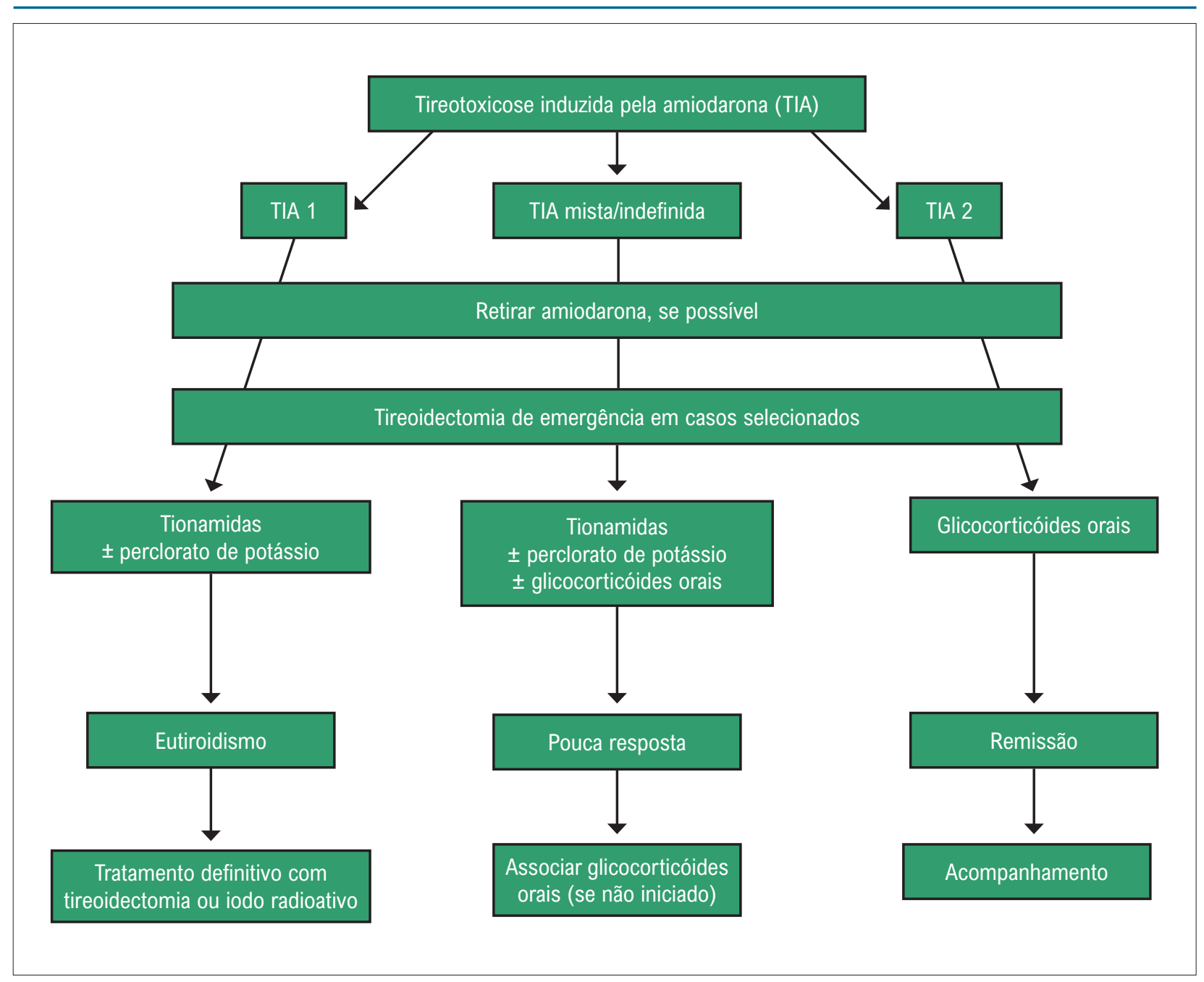

Figura 2 - Algoritmo para manejo de tireotoxicose induzida pela amiodarona (TIA). Modificado de Bartalena L, et al. ${ }^{14}$

pacientes cardiopatas, o tratamento deve ser instituído para que o eutireoidismo seja atingido mais rapidamente. ${ }^{14,19,36}$ A decisão de tratar casos leves ou subclínicos deve ser feita levando em consideração as alterações cardíacas do paciente. $^{14}$

Tem sido sugerido que, em casos bem definidos de TIA 2, o tratamento com corticosteroides é mais efetivo do que o tratamento com DAT. ${ }^{29,37}$ As doses utilizadas são de 30-40mg/dia de prednisona ou dose equivalente de outro glicocorticoide por dois a três meses, com posterior retirada gradual baseada na resposta clínico. ${ }^{14,24} \mathrm{Em}$ casos graves, assim como nos casos de TIA 1 e mista/indefinida, a tireoidectomia radical deve ser considerada. . $^{814,38}$

\section{Tratamento: TIA mistas ou indefinidas}

Formas mistas ou indefinidas ainda não estão completamente caracterizadas. Acredita-se que esses casos envolvam mecanismos patogênicos mistos dos dois subtipos, tanto de aumento da produção hormonal quanto por tireoidite destrutiva. ${ }^{14,36}$
O tratamento das formas mistas ou indefinidas deve ser feito com DAT, podendo ser associados corticosteroides orais no início do tratamento, ou após 4-6 semanas se a resposta for pequena. ${ }^{14,19,35} \mathrm{Em}$ casos mais severos, a terapia combinada com DAT e corticosteroides deve ser iniciada prontamente. ${ }^{24}$

\section{Tratamento: Tireoidectomia}

À tireoidectomia total corresponde à melhor opção em pacientes cujo tratamento clínico é falho ou naqueles em que há demora de resposta terapêutica associada a função ventricular deprimida, sendo a melhor alternativa para restauração imediata do eutireoidismo. ${ }^{14,39,40}$ Apesar dos riscos associados à tireoidectomia, esta deve ser considerada antes que o paciente evolua com piora clínica severa, pois a demora na indicação da cirurgia está associada ao aumento da mortalidade..$^{24,39-42}$ Diversos estudos avaliando pacientes com TIA submetidos à tireoidectomia relataram baixa morbidade associada ao procedimento, apresentando mortalidade de $0 \%$ a $1.9 \%{ }^{40-44}$

Um estudo observacional recente, 207 pacientes com TIA (57 tireoidectomizados, 156 tratamento clínico), evidenciou 
menor mortalidade nos pacientes submetidos a tireoidectomia comparada aos tratados apenas clinicamente, particularmente em pacientes com FEVE $<40 \%$. Neste mesmo estudo foi demonstrada uma melhora significativa da FEVE após a restauração do eutireoidismo, sendo mais evidente nos pacientes com FEVE $<40 \% .{ }^{42}$ Outros três estudos também relataram melhora significativa da função cardíaca após tireoidectomia, sendo três pacientes retirados da lista de transplante cardíaco após restauração do eutireoidismo. ${ }^{40,41,43}$

Se a tireoidectomia total for considerada, a avaliação individualizada dos riscos e benefícios deve ser feita, e a decisão deve ser multidisciplinar, envolvendo cardiologistas, endocrinologistas, cirurgiões e anestesistas. É imprescindível que um cirurgião com alto volume operatório e experiência com tireoidectomias seja o responsável pelo procedimento. ${ }^{14}$

Tireoidectomia total deve ser considerada quando há: $14,24,39,43$ :

- Resposta insuficiente ao tratamento medicamentoso com DAT e corticosteroides;

- Deterioração rápida da função cardíaca;

- Doença cardíaca avançada, displasia arritmogênica ventricular direita, e arritmias malignas;

- Tratamento definitivo alternativo ao radioiodo;

\section{Conclusão}

Dadas as consequências acarretadas pela TIA, salientase a importância de diagnosticar e tratar os subtipos de TIA conjuntamente. Enfatiza-se a importância de as decisões terapêuticas serem tomadas de forma conjunta

\section{Referências}

1. Martino E, Bartalena L, Bogazzi F, Braverman LE. The effects of amiodarone on the thyroid. Endocr Rev.2001;22(2): 240-54.

2. Peter T, Hamer A, Mandel WJ, Weiss D. Evaluation of amiodarone therapy in the treatment of drug-resistant cardiac arrhythmias: Long-term follow-up. Am Heart J.1983;106(4):943-50. doi:10.1016/0002-8703(83)90020-0

3. Claro J, Candia R, Rada G, Baraona F, Larrondo F, Letelier LM. Amiodarone versus other pharmacological interventions for prevention of sudden cardiac death. Cochrane Database of Systematic Reviews. 2015;12:No CD008093. DOI: 10.1002/14651858. CD008093.pub2

4. Trip MD, Wiersinga W, Plomp TA. Incidence, predictability, and pathogenesis of amiodarone-induced thyrotoxicosis and hypothyroidism. Am J Med. 1991;91(5):507-11. doi:10.1016/0002-9343(91)90187-3

5. Yiu KH, Jim MH, Siu CW, Lee $\mathrm{CH}$, Yuen M, Mok M, et al. Amiodaroneinduced thyrotoxicosis is a predictor of adverse cardiovascular outcome. J Clin Endocrinol Metab. 2009;94(1):109-14.

6. Benjamens S, Dullaart RPF, Sluiter WJ, Rienstra M, van Gelder IC, Links TP. The clinical value of regular thyroid function tests during amiodarone treatment. Eur J Endrocrinol. 2017; 177(1):9-14

7. Martino E, Aghini-Lombardi F, Mariotti S, Bartalena L, Lenziardi M, Ceccarelli $\mathrm{C}$, et al. Amiodarone iodine-induced hypothyroidism: Risk factors and follow-up in 28 cases. Clin Endocrinol. 1987;126(2):227-37. doi: 10.1111/ j.1365-2265.1987.tb00781.x

8. Martino E, Safran M, Aghini-Lombardi F, Rajatanavin R, Lenziardi M, Fay $M$, et al. Environmental lodine Intake and Thyroid Dysfunction During por cardiologistas e endocrinologistas, e que nos casos mais severos a tireoidectomia deve ser considerada antes que ocorra piora clínica exagerada.

Estudos clínicos envolvendo pacientes com TIA ainda são limitados e insuficientes, principalmente ensaios clínicos randomizados multicêntricos. Visto que a amiodarona é uma droga bastante utilizada, e devido as consequências da TIA, destaca-se a necessidade de novos ensaios clínicos para aprimorar o manejo destes pacientes.

\section{Contribuição dos autores}

Concepção e desenho da pesquisa, Redação do manuscrito e Revisão crítica do manuscrito quanto ao conteúdo intelectual importante: Souza LVF, Campagnolo MT, Martins LCB, Scanavacca MI; Obtenção de dados, Análise e interpretação dos dados: Souza LVF, Campagnolo MT, Martins LCB.

\section{Potencial conflito de interesse}

Não há conflito com o presente artigo

\section{Fontes de financiamento}

O presente estudo não teve fontes de financiamento externas.

\section{Vinculação acadêmica}

Não há vinculação deste estudo a programas de pósgraduação.

Chronic Amiodarone Therapy. Ann Intern Med. 1984101(1):28-34. doi 10.7326/0003-4819-101-1-28

9. Lee KF1, Lee KM, Fung TT. Amiodarone-induced thyroid dysfunction in the Hong Kong Chinese population. Hong Kong Med J. 2010 Dec;16(6):434-9.

10. Barrett B, Hawkes CP, Isaza A, Bauer AJ. The Effects of Amiodarone on Thyroid Function in Pediatric and Young Adult Patients. J Clin Endocrinol Metab. 2019 Nov;104(11):5540-6. doi: 10.1210/jc.2019-00990

11. Uchida T, Kasai T, Takagi A, Sekita G, Komiya K, Takeno K, et al. Prevalence of Amiodarone-Induced Thyrotoxicosis and Associated Risk Factors in Japanese Patients. Int J Endocrinol.2014;2014:1-6. doi:10.1155/2014/534904

12. Huang CJ, Chen PJ, Chang JW, Huang DF, Chang SL, Chen SA, et al. Amiodarone-induced thyroid dysfunction in Taiwan: a retrospective cohort study. Int J Clin Pharmacy. 2014;36(2):405-11 doi:10.1007/s11096-0139910-9

13. Bogazzi F, Tomisti L, Bartalena L, Aghini-Lombardi F, Martino E. Amiodarone and the thyroid: a 2012 update. J Endocrinol Investig. 2012;35(3):340-8. doi: $10.3275 / 8298$

14. Bartalena L, Bogazzi F, Chiovato L, Hubelewska-Dydejczyk A, Links TP Vanderpump M. 2018 European Thyroid Association (ETA) guidelines for the management of amiodarone-associated thyroid dysfunction. Eur Thyroid J. $2018 ; 7(2): 55-66$.

15. O'Sullivan AJ, Lewis M, Diamond T: Amiodarone-induced thyrotoxicosis: left ventricular dysfunction is associated with increased mortality. Eur J Endocrinol. 2006;154(4):533-6. 
16. World Health Organization. (WHO) lodine deficiency in Europe: a continuing public health problem. [Internet] Available from: http://www. who.int/iris/handle/10665/43398

17. Ross IL, Marshall D, Okreglicki A, Isaacs S, Levitt NS. Amiodarone-induced thyroid dysfunction. S Afr Med J. 2005;95(3):180-3.

18. Pavan R, Jesus AMX, Maciel LMZ. A amiodarona e a tireóide. Arq Bras Endocrinol Metab. 2004; 48(1):176-82.

19. Maia AL, Scheffel RS, Meyer ELS, Carvalho GA, Graf H, et al. Consenso Brasileiro para o diagnóstico e tratamento do hipertireoidismo: recomendações do Departamento de Tireoide da Sociedade Brasileira de Endocrinologia e Metabologia (SBEM). Arq Bras Endocrinol Metab. 2013;57(3):205-32.

20. Bogazzi F, Bartalena L, Gasperi M, Braverman LE, Martino E. The various effects of amiodarone on thyroid function. Thyroid. 2001;11(5)511-9. doi: 10.1089/105072501300176471

21. Elnaggar MN, Jbeili K, Nik-Hussin N, Kozhippally M, Pappachan JM. Amiodarone-Induced Thyroid Dysfunction: a clinical Update. Exp Clin Endocrinol Diabetes. 2018 ; 126(6):333-41. doi: 10.1055/a-0577-7574.

22. Rao RH, McCready VR, Spathis GS. Iodine kinects studies during Amiodarone treatment. J Clin Endocrinol Metab. 1986; 62(3):563-8.

23. Tavares MB, Motta PRV, Barros VF, Cezana C, Ferreira LB, Saar SMA, Brandão CDG. Distúrbios da função tireoidiana induzidos pela amiodarona. Sales J Health Sci. 2016;2(2):39-47.

24. Ross DS, Burch HB, Coorper DS, Greenlee MC, Laurberg P, Maia AL, et al. 2016 American Thyroid Association (ATA) guidelines for diagnosis and management of hyperthyroidism and other causes of thyroxicosis. Thyroid. 2016;26(10:1343-421. Doi: 10.1089/thy.2016.0229.

25. Stan, M N, Ammash N M, Warnes C A, Brennan M D, Thapa P, et al. Body mass index and the development of amiodarone-induced thyrotoxicosis in adults with congenital heart disease-A cohort study. Int J Cardiol. 2013;167(3):821-6. doi:10.1016/j.ijcard.2012.02.015

26. Trip MD, Wiersinga W, Plomp TA: Incidence, predictability, and pathogenesis of amiodarone-induced thyrotoxicosis and hypothyroidism. Am J Med .1991;91(5):507-11.

27. Eaton SEM, Euinton HA, Newman CM, Weetman AP, BennetWM. Clinical experience of amiodarone-induced thyrotoxicosis over a 3-year period: role of colour-flow Doppler sonography. Clin Endocrinol. 2002;56(1):33-8. doi:10.1046/j.0300-0664.2001.01457.x

28. Bogazzi F, Martino E, Dell'Unto E, Brogioni S, Cosci C, Aghini-Lombardi F, et al. Thyroid color flow Doppler sonography and radioiodine uptake in 55 consecutive patients with amiodarone-induced thyrotoxicosis. J Endocrinol Invest .2003;26(7):635-40.

29. Eskes SA, Endert E, Fliers E, GerskusRB, Dullaart RP, Links TP, et al. Treatment of amiodarone-induced thyrotoxicosis type 2 : a randomized clinical trial. J Clin Endocrinol Metab. 2012;87(2):499-506.

30. Uzan L, Guignat L, Meune C, Mouly S, Weber S, Bertagna X, et al. Continuation of amiodarone therapy despite type II amiodarone-induced thyrotoxicosis. Drug Saf. 2006;29(3):231-6.
31. Bogazzi F, Bartalena L, Tomisti L, Rossi G, Brogioni S, Martino E: Continuation of amiodarone delays restoration of euthyroidism in patients with type 2 amiodarone-induced thyrotoxicosis treated with prednisone: a pilot study. J Clin Endocrinol Metab. 2011;96(11): 3374-80.

32. Sato K, Shiga T, Matsuda N, Onoda N, Takano K, Hagiara N, Kasanuki H: Mild and short recurrence of type II amiodarone-induced thyrotoxicosis in three patients receiving amiodarone continuously for more than 10 years. Endocrine J 2006;53(4):531-8.

33. Maqdasy S, Batisse-Lignier, M, Auclair C, Desbiez F, Citron B, Thieblot, $\mathrm{P}_{\text {, }}$ et al. Amiodarone-Induced Thyrotoxicosis Recurrence After Amiodarone Reintroduction. Am J Cardiol.2016; 117(7), 1112-6. doi:10.1016/j. amjcard.2016.01.003

34. Stan, M, Sathananthan M, Warnes $C$, Brennan M, Thapa P, Bahn R. Amiodarone-Induced Thyrotoxicosis in Adults with Congenital Heart Disease - Clinical Presentation and Response to Therapy. Endocr Pract.2014;20(1):33-40. doi:10.4158/ep13059.

35. Bartalena L, Brogioni S, Grasso L, Bogazzi F, Burelli A, Martino E. Treatment of amiodarone-induced thyrotoxicosis, a difficult challenge: results of a prospective study. J Clin Endocrinol Metab. 1996;81(8):2930-3.

36. Bogazzi F, Bartalena L, Martino E. Approach to the patient with amiodaroneinduced thyrotoxicosis. J Clin Endocrinol Metab. 2010;95 2529-35.

37. Bogazzi F, Tomisti L, Rossi G, Dell'Unto E, Pepe P, Bartalena L, Martino E: Glucocorticoids are preferable to thionamides as first-line treatment for amiodarone-induced thyrotoxicosis due to destructive thyroiditis: a matched retrospective cohort study. J Clin Endocrinol Metab. 2009;94(10):3757-62.

38. Isaacs M, Costin M, Bova R, Barrett HL, Heffernan D, Samaras K, et al. Management of Amiodarone-Induced Thyrotoxicosis at a Cardiac Transplantation Centre. Front Endocrinol. 2018:482. doi: 10.3389/ fendo.2018.00482.

39. Houghton SG, Farley DR, Brennan MD, van Heerden JA, Thompson GB, Grant CS. Surgical management of amiodarone-associated thyrotoxicosis: Mayo Clinical Experience. World J Surg.2004; 28(11):1083-7.

40. Tomisti L, Materazzi G, Bartalena L, Rossi G, Marchello A, Moretti M, et al. Total thyroidectomy in patients with amiodarone-induced thyrotoxicosis and severe left ventricular systolic dysfunction. J Clin Endocrinol Metab. 2012;97(10)::3515-21.

41. Kaderli RM, Fahrner R, Christ ER, Stettler C, Fuhrer J, Martinelli M, et al. Total thyroidectomy for amiodarone-induced thyrotoxicosis in the hyperthyroid state. Exp Clin Endocrinol Diabetes. 2016;124(1):45-8.

42. Cappellani D, Papini P, Pingitore A, Tomisti L, Mantuano M, Di Certo AM, et al. Comparison Between Total Thyroidectomy and Medical Therapy for Amiodarone-Induced Thyrotoxicosis. J Clin Endocrinol Metab. 2020 Jan 1;105(1):242-51.

43. Gough J, Gough IR. Total thyroidectomy for amiodarone-associated thyrotoxicosis in patients with severe cardiac disease. World J Surg. 2006;30(11):1957-61.

44. Patel N, Inder W J, Sullivan C, Kaye G. An Audit of Amiodarone-induced Thyrotoxicosis - do Anti-thyroid Drugs alone Provide Adequate Treatment? Heart, Lung Circ. 2014;23(6):549-54. doi:10.1016/j.hlc.2014.01.013 